\title{
10. A PROPÓSITO DE ALGUNAS CONFUSIONES EN TORNO A CLADANTHUS SCARIOSUS (BALL) OBERPR. \& VOGT (ASTERACEAE)
}

\author{
Francisco GÓMIZ GARCÍA
}

About some confusion regarding Cladanthus scariosus (Ball) Oberpr. \& Vogt (Asteraceae)

Palabras clave. Cladanthus, Chamaemelum, Ormenis, Santolina, Marruecos, Norte de África.

Key words. Cladanthus, Chamaemelum, Ormenis, Santolina, Morocco, North Africa.

En la bibliografía de los últimos veinte años se ha podido observar, en más de una ocasión, el deslizamiento de ciertos errores o equivocaciones en torno a Cladanthus scariosus (Ball) Oberpr. \& Vogt -en particular su confusión con Ormenis africana (A. J. Jord. \& Fourr.) Litard. \& Maire (sinónimo de Santolina africana A. J. Jord. $\&$ Fourr., nombre en uso)-, alguno de los cuales va a ser puesto de manifiesto, apuntando a su posible causa. Sin embargo, como ambos táxones han experimentado a lo largo de su historia numerosas combinaciones y, según el autor y el momento de que se trate, pueden encontrarse incluidos en diferentes géneros, parece adecuado, antes de proseguir, presentar sendos esquemas sinonímicos que faciliten la comprensión de los comentarios que se expondrán a continuación.

Cladanthus scariosus (Ball) Oberpr. \& Vogt, Willdenowia 32: 197 (2002).

三Santolina scariosa Ball, J. Bot. 11: 365

(1873), (basiónimo).

= Ormenis heterophylla Coss., Bull. Soc. Bot. France 22: 61 (1875), nomen nudum. = Anthemis heterophylla Coss. ex Ball, J. Linn. Soc., Bot. XVI: 507 (1878).

$\equiv$ Ormenis scariosa (Ball) Litard. \& Maire, Bull. Soc. Hist. Nat. Afrique N. 14: 93 (1923).

इChamaemelum scariosum (Ball) Benedí, Candollea 43: 126 (1988).
Santolina africana A. J. Jord. \& Fourr., Icon. F1. Eur. II. 8 (1869).

三Ormenis africana (A. J. Jord. \& Fourr.) Litard. \& Maire in Jahandiez \& Maire, Cat. Pl. Maroc, III. 765 (1934).

$\equiv$ Santolina chamaecyparissus L. var. africana (A. J. Jord. \& Fourr.) Guinea, Anales Inst. Bot. Cavanilles 27: 42 (1970).

= Ormenis pseudo-santolina Maire, Mém. Soc. Sci. Nat. Maroc 15: 41 (1926).

Es posible que la transferencia de Santolina africana al género Ormenis (Cass.) Cass., hecha por Litardiere y Maire (loc. cit.) basándose en ciertos caracteres carpológicos como la presencia de células mixógenas propias del género Chamaemelum (Oberprieler, 2002: 257)-, haya sido la causa de frecuentes confusiones, algunas de las cuales han llegado hasta nuestros días. Recientemente, Benabid (2000: 208) y Charco (2001: 581) ilustran como Ormenis africana lo que no es otra cosa que Cladanthus scariosus. En el primero de los casos, esto resulta evidente -debido a la falta de detalle de la fotografía- sólo a quien conozca en vivo la planta de Ball; en el segundo, por el contrario -perfectamente ilustrado con dos buenas fotografías-, queda meridianamente claro que no se trata de la planta que se dice que es.

El problema de las combinaciones entre 
Santolina L. y Ormenis viene de finales del siglo XIX, como puede leerse en el primer párrafo del comentario a Chamaemelum scariosum que hace Benedí (1988:126), y continuaron en el siglo XX. Battandier (1921: 8) desestimó la presencia de lígulas en este endemismo marroquí (Cladanthus scariosus) y, siguiendo el criterio original de Ball, aceptó a la planta en el género Santolina reconociendo el binomen del autor irlandés. Litardière y Maire (1930: 20), después de haber llevado siete años antes la planta de Ball al género Ormenis (loc. cit.), siguen dándole vueltas al asunto y crean el subgénero Santolinopsis -que dejan sin describir- para incluir en él a Ormenis scariosa y a Ormenis eriolepis Coss. ex Maire.

Es posible que este accidentado vaivén entre Ormenis y Santolina haya llevado en diversas ocasiones a confundir estas plantas, muy diferentes entre sí. Confusión que no ha contribuido a aclarar Benedí (loc. cit.) quien, centrando más su trabajo en los aspectos carpológicos de Chamaemelum Mill., da incomprensiblemente por anual a la especie más leñosa del género -suffruticosa, según Ball (sub Anthemis heterophylla, loc. cit.)-. Lo hace en dos ocasiones: una de forma explícita, en el segundo párrafo del comentario a Chamaemelum scariosum- "Ch. scariosum es próximo a Ch. eriolepis (Coss. ex Maire)Benedí, al que se asemeja por ser planta anual..."-; y otra, de forma implícita, al excluirla de las perennes, en el primer y único párrafo del comentario a Chamaemelum flahaultii (Emb.)Benedí -"Se trata, junto con Ch. nobile (L.)All., de las únicas especies perennes dentro del género Chamaemelum."-.

Aunque de problemática clasificación, debido a la mencionada presencia, en su delgado pericarpo, de células mixógenas ausentes en otras especies de Santolina que presentan grueso pericarpo esclerenquimatoso- la planta combinada como Ormenis africana debe permanecer en el género Santolina tal y como se percibe al primer golpe de vista, cuando se la tiene delante, y tal y como establecieron desde sus descriptores, Jordan y Fourreau, hasta el último autor que, según nuestro conocimiento, la recombinó dentro de dicho género: Guinea (1970: 42). Sus capítulos discoideos y sus hojas, son, inequívocamente, los de una Santolina; y no debe confundirse, en ningún caso, con Cladanthus scariosus por más que ambas se presenten bajo la forma de subarbustos densos (figs. 4 y 5). A continuación se presentan las diferencias más importantes existentes entre ambos géneros (tab. 1).

\begin{tabular}{ll}
\hline \multicolumn{1}{c}{ Santolina } & \multicolumn{1}{c}{ Cladanthus } \\
\hline Arbustillos. & Hierbas anuales o perennes, o subarbustos. \\
Capítulos discoideos. & Capítulos radiados. \\
Receptáculo ligeramente convexo. & Receptáculo cónico o elongado. \\
Páleas no carinadas. & Páleas fuertemente car inadas. \\
Cipselas oblongas, 3-5-angulosas. & Cipselas obovadas, ligeramente aplanadas. \\
Pericarpo generalmente esclerenquimatoso. & Pericarpo con células mixógenas. \\
Disco apical horizontal y estilopodio central. & Disco apical oblicuo y estilopodio sublateral. \\
\hline
\end{tabular}

Tabla 1. Principales diferencias entre los géneros Santolina y Cladanthus. Main differences between genera Santolina and Cladanthus. 


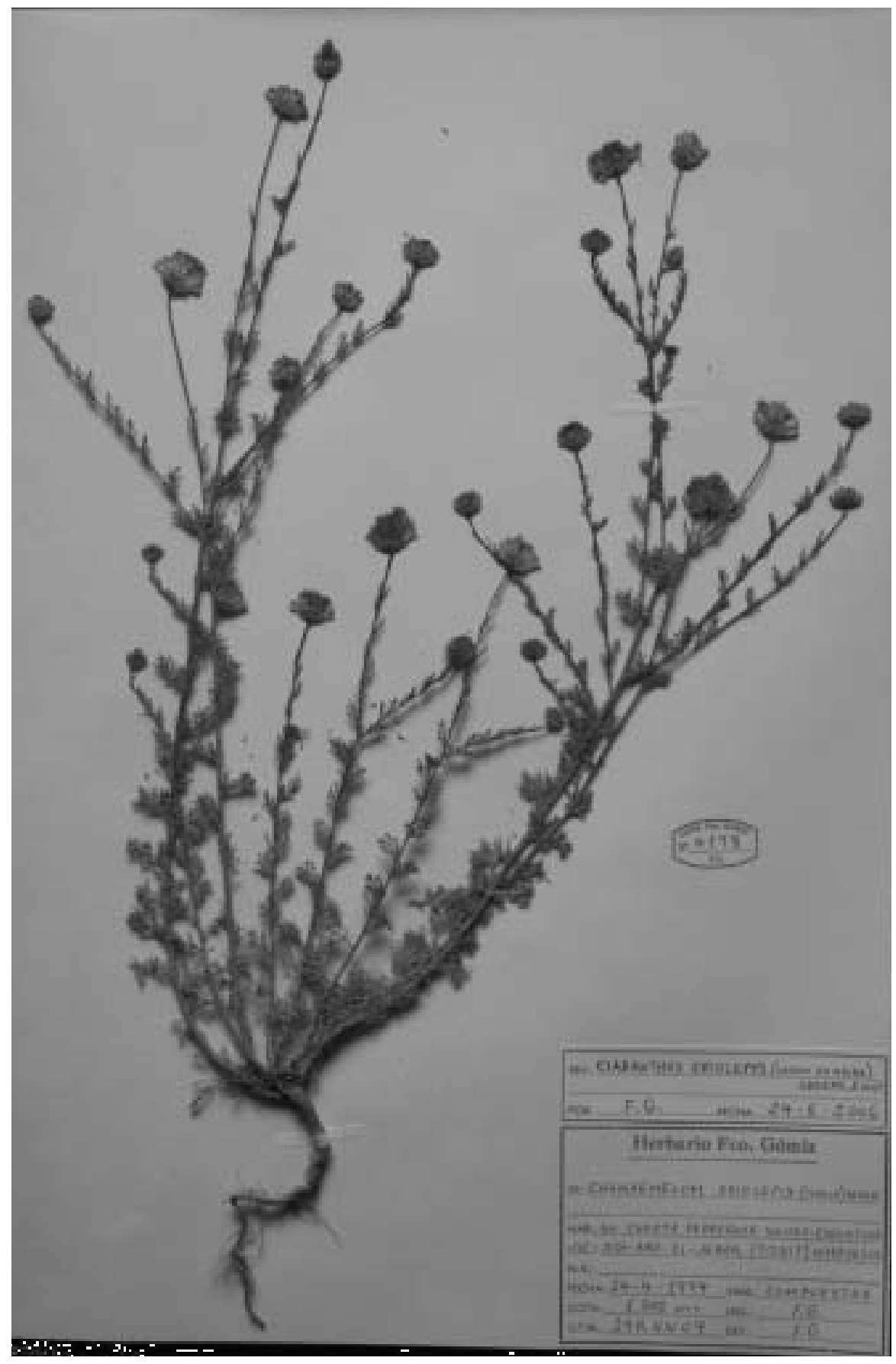

Figura 1. Cladanthus eriolepis (Coss. ex Maire) Oberpr. \& Vogt. 


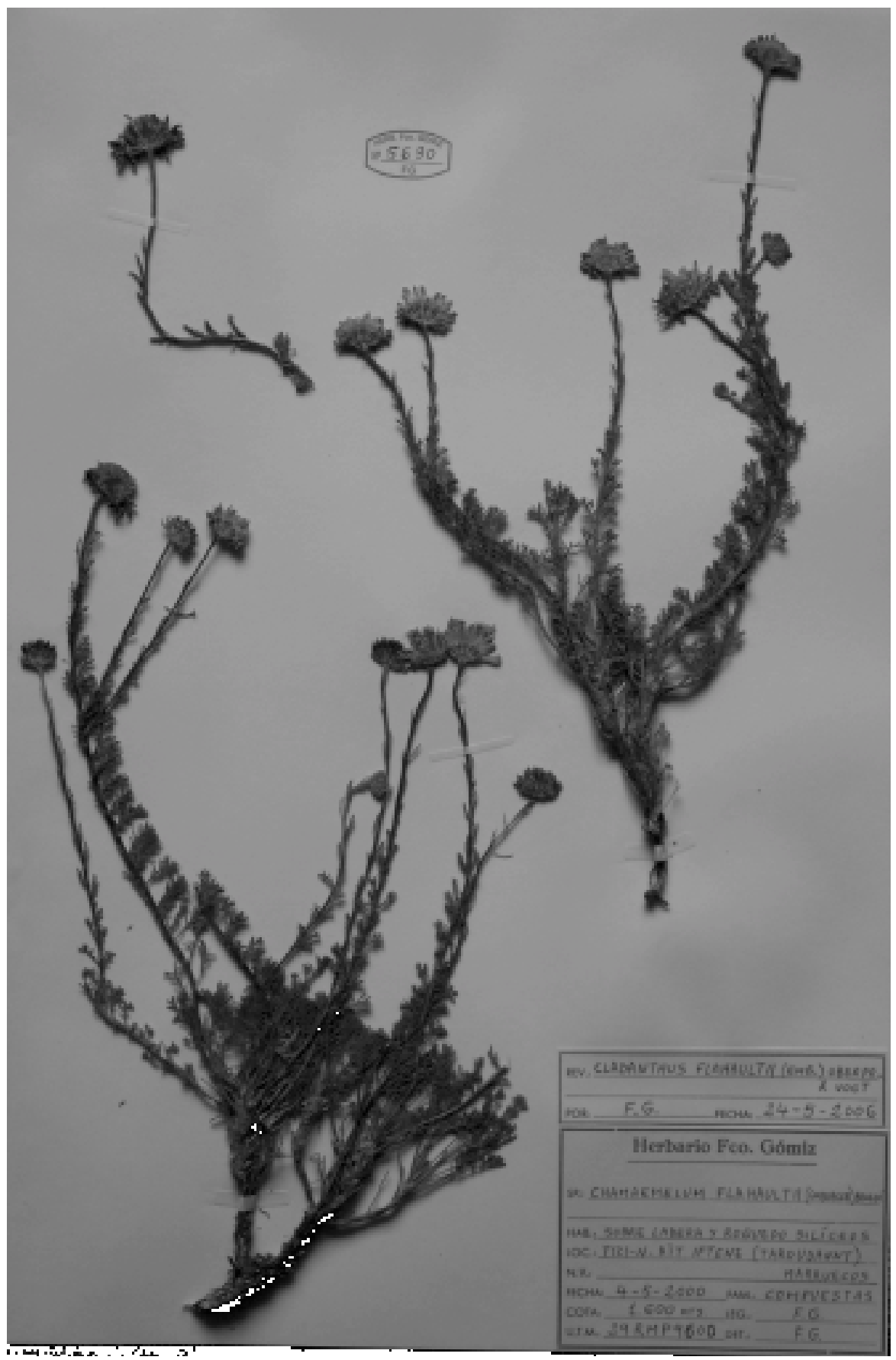

Figura 2. Cladanthus flahaultii (Emb.) Oberpr. \& Vogt. 


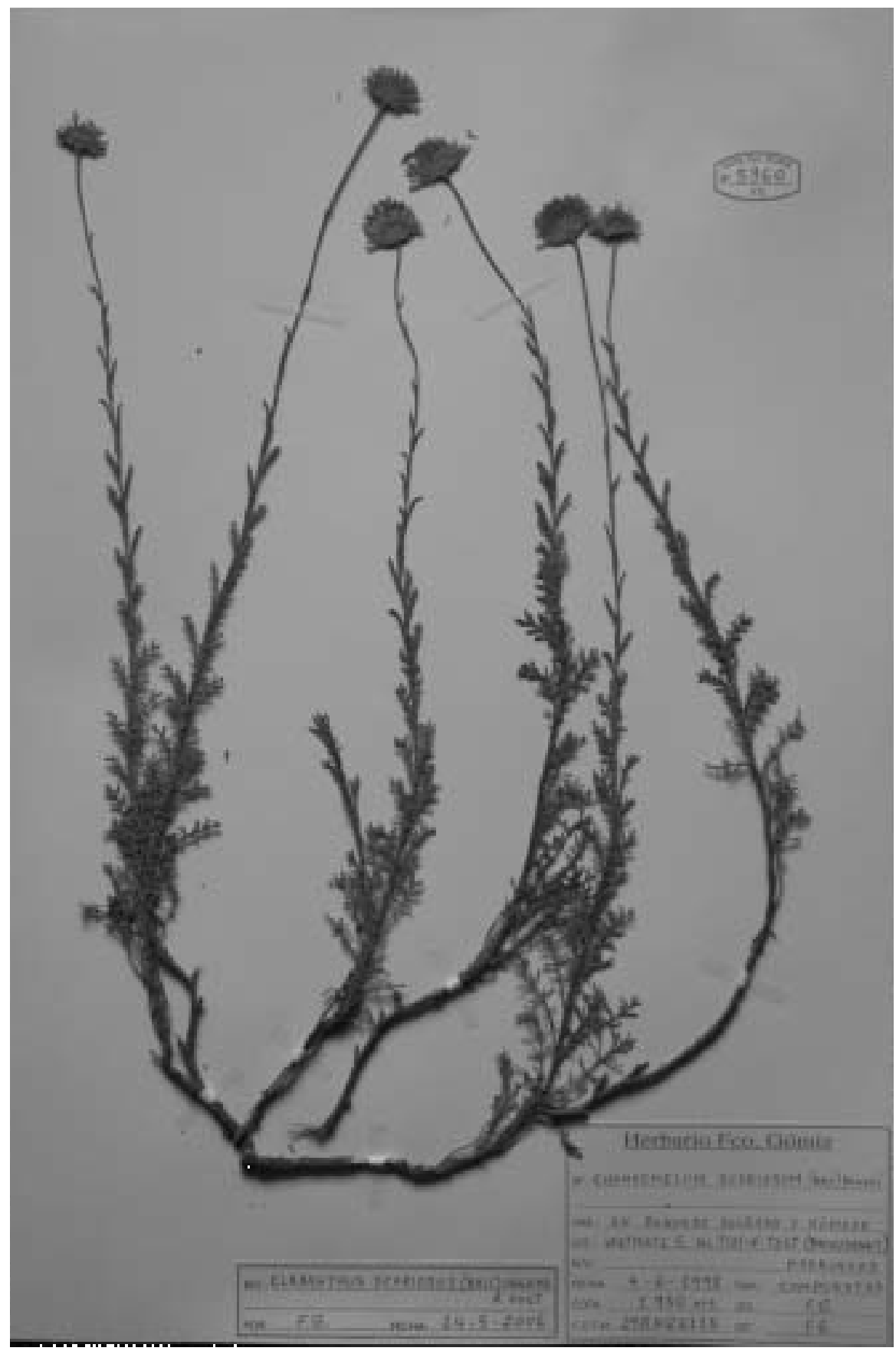

Figura 3. Cladanthus scariosus (Ball) Oberpr. \& Vogt. 


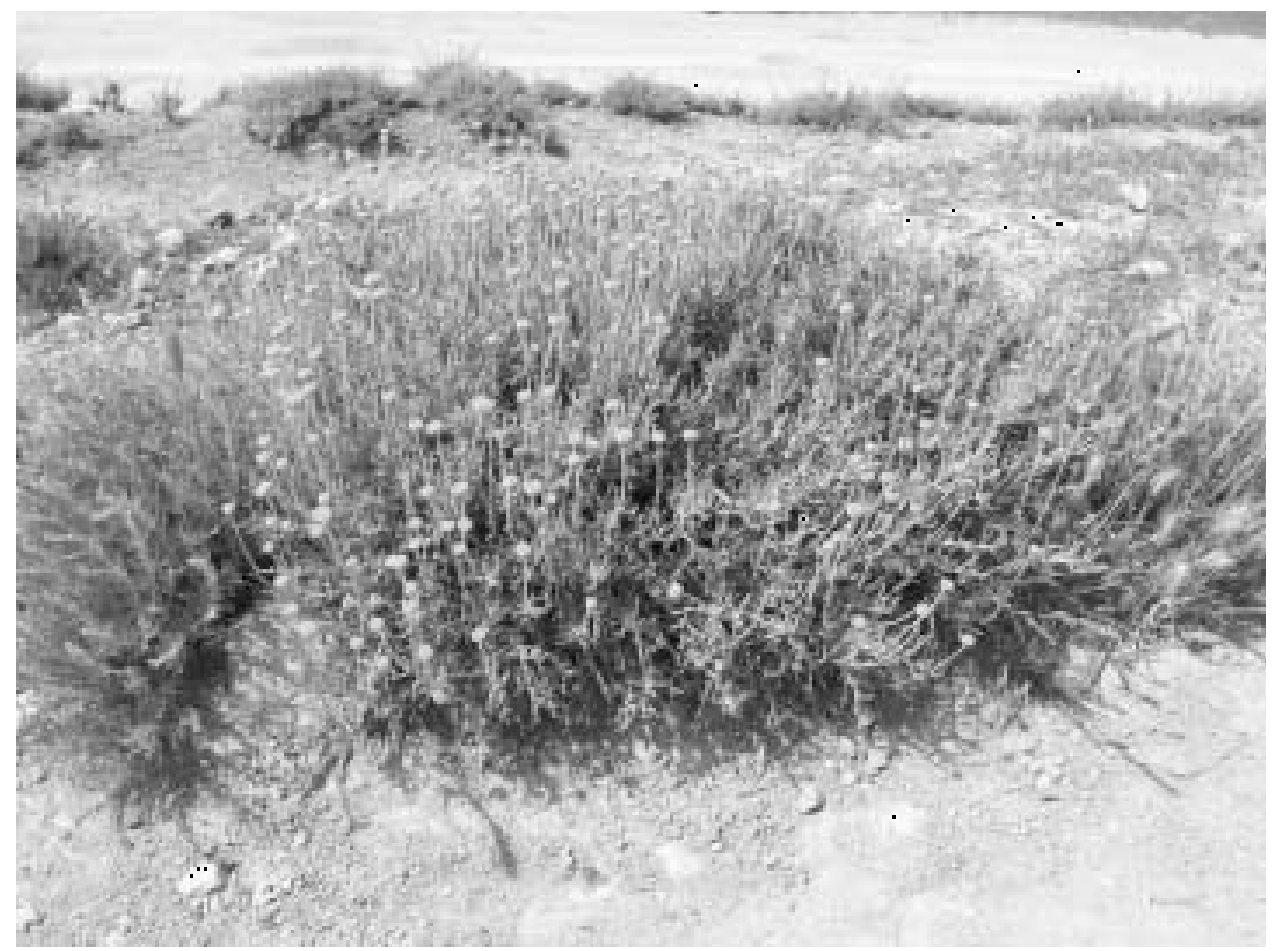

Figura 4. Santolina africana A. J. Jord. \& Fourr.

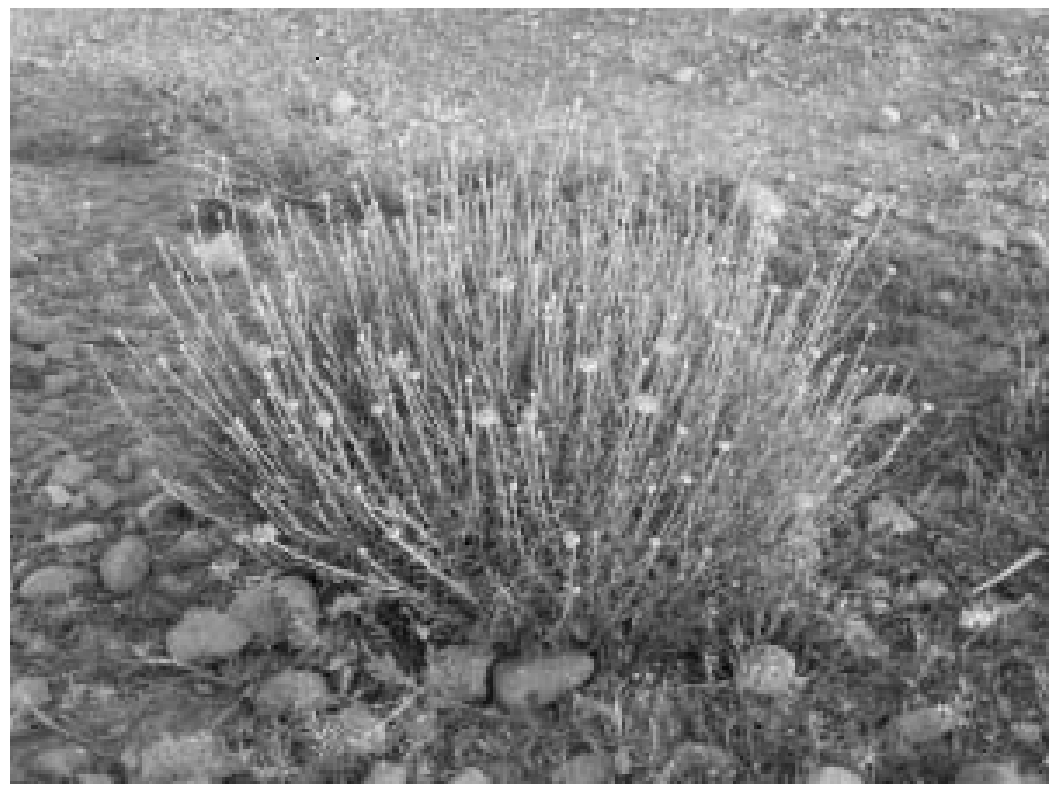

Figura 5. Cladanthus scariosus (Ball) Oberpr. \& Vogt. 
Otras especies del mismo género presentes en Marruecos son: Santolina adscensionis Sennen ex Maire y Santolina rosmarinifolia L. que presenta dos subespecies, subsp. canescens (Lag.) Nyman y subsp. pectinata (Lag.) Maire; pero su estudio no es objeto de la presente nota.

Por lo que respecta a Cladanthus género caracterizado por sus cipselas con disco apical oblicuo y estilopodio sublateral; brácteas interseminales fuertemente carinadas; flores radiadas de parcial a totalmente amarillas o anaranjadas; y hojas desde dentadas a bipinnatisectas (Oberprieler, 2002: 263)-; partiendo, por un lado, del material existente en mi herbario personal -consultable a través del herbario LEB- así como del disponible en los herbarios BC y MA; y, por otro, de la bibliografía consultada -con especial atención a los trabajos de Benedí-, se ha elaborado una clave para la determinación de las especies marroquíes de dicho género. En la misma ha sido incluido C. mixtus como planta anual, tal y como hace Benedí (1987: 58) para las especies ibéricas de Chamaemelum, a pesar de que, como este mismo autor indica (Benedí, 1986: 4; 1987 : 62), esta especie es extremadamente polimorfa en lo que se refiere, entre otras cosas, a su porte y ramificación. De hecho, entre la sinonimia de la especie se incluye Ormenis multicaulis Braun-Blanq. \& Maire, Bull. Soc. Hist. Nat. Afrique N. 16: 35 (1925), que sus autores describieron como bienal y de base más o menos sufruticosa. Convendrá, pues, para la correcta determinación de las plantas pertenecientes a esta especie, tomar en consideración, además de su ciclo biológico, otros caracteres que le son propios; como, por ejemplo, sus características hojas dentadas o cortamente divididas.

Por su valor corológico se relacionan al final, los pliegos existentes en los tres herbarios mencionados, correspondientes a las tres especies endémicas marroquíes pertenecientes a dicho género: $C$. eriolepis (Coss. ex Maire) Oberpr. \& Vogt, $C$. flahaultii (Emb.) Oberpr. \& Vogt y $C$. scariosus. (Figs. 1, 2 y 3).

\section{Claves}

1. Plantas anuales ............................................. 2

1. Plantas perennes .................................................. 4

2. Capítulos sésiles C. arabicus

2. Capítulos pedunculados 3

3. Receptáculo cilíndrico en la fructificación; brácteas interseminales encerrando al ovario; base del tubo de las flores flosculosas calcariforme, con espolón basal de c. $1 \mathrm{~mm}$

C. mixtus

3. Receptáculo hemisférico-subcónico en la fructificación; brácteas interseminales no encerrando al ovario; base del tubo de las flores flosculosas caliptriforme, con dos gibas basales casi opuestas

C. eriolepis

4. Planta con ramillas postrado-ascendentes, de base herbácea, con densos grupos de hojas basales; base del tubo de las flores flosculosas eroso-caliptriforme; limbo de las flores hemiliguladas con banda rojiza por la cara posterior

C. flahaultii

4. Planta con ramillas arqueado-erectas, de base leñosa, sin densos grupos de hojas basales; base del tubo de las flores flosculosas oblicuo-caliptriforme; limbo de las flores hemiliguladas amarillo por ambas caras .......

C. scariosum

1. Annual herbs ............................................... 2

1. Perennial plants ........................................... 4

2. Capitula sessile C. arabicus

2. Capitula pedunculate 3

3. Receptacle cylindrical, elongated in maturity; ovaries completely enclosed in paleae; base of tubular florets calcariform with adaxial spur c. $1 \mathrm{~mm}$ (sometimes biennial or subperennial)

C. mixtus

3. Receptacle hemispherical-subconical in maturity; ovaries not completely enclosed in paleae; base of tubular florets calyptriform with two basal almost opposed humps

C. eriolepis 
4. Perennial herb with dense groups of basal leaves; base of tubular florets erosecalyptriform; rays with a reddish stripe beneath

C. flahaultii

4. Half-shrub without dense groups of basal leaves; base of tubular florets obliquecalyptriform; rays yellow on both sides

C. scariosum

\section{Material examinado}

\section{Cladanthus eriolepis}

Marruecos. Antiatlas: Içafen, in lapidosis arenaceis, 1200 m, 19-IV-1931, R. Maire, BC 144111; Agadir: prope oppidulum Tafraoute, Tizi Mlil, ad $1200 \mathrm{~m}$, in dumosis raris secus viam, solo siliceo, 9358, 26-V-1985, C. Blanché, J. Fdez. Casas, J. Molero, J. M. Montserrat \& A. Romo, MA 340265; Sidi Abd el Jbar, cuneta pedregosa silíceo-esquistosa, $29^{\circ} 46^{\prime} \mathrm{N} 8^{\circ} 55^{\prime} \mathrm{W}$, 29RNN09, 1300 m, 24-IV-1994, Gómiz, Herb. F. Gómiz 4198; Vertiente N del Tizi Bachkoum, roquedo vertical eruptivo, $30^{\circ} 41^{\prime} \mathrm{N} 7^{\circ} 16^{\prime} \mathrm{W}$, 29RPP69, 1525 m, 8-V-1995, Gómiz, Herb. F. Gómiz 4479. Vertiente S del Tizi n'Tazazert (J. Sarhro), en borde pedregoso de pista, $1825 \mathrm{~m}$, $31^{\circ} 05^{\prime} 34^{\prime \prime} \mathrm{N} \mathrm{5} 5^{\circ} 46^{\prime} 38^{\prime \prime} \mathrm{W}, 30 \mathrm{RTV} 3543,16-\mathrm{V}-$ 2006, Gómiz, Herb. F. Gómiz 7436. Draâ: In lapidosis arenaceis montium Bani, circa Foumel-Hassan, 500-600 m, 5-IV-1935, R. Maire \& E. Wilczek, BC 88275. Unos $14 \mathrm{~km}$ al NE de Ouarzazate, cuneta pedregosa, $30^{\circ} 59^{\prime} \mathrm{N} 6^{\circ} 49^{\prime}$ W, 29RQQ02, 1135 m, 10-V-1995, Gómiz, Herb. F. Gómiz 4512; Inmediaciones de Ait Ouabelli, en cuneta pedregosa, $29^{\circ} 14$ ' $47^{\prime \prime} \mathrm{N} 8^{\circ} 30^{\prime} 29^{\prime}$ " W, 29RNN4735, 470 m, 4-IV-1996, Gómiz, Herb. F. Gómiz 4741. Marruecos desértico oriental: Unos $7 \mathrm{~km}$ al NE del cruce del oued Zerzel con la ctra. P-21, en ladera rocosa caliza orientada al $\mathrm{N}, 950 \mathrm{~m}, 31^{\circ} 35^{\prime} 30^{\prime}, \mathrm{N} \mathrm{4}{ }^{\circ} 07^{\prime} 33^{\prime}, \mathrm{W}$, 30RUV9395, 16-V-2006, Gómiz, Herb. F. Gómiz 7435.

\section{Cladanthus flahaultii}

Marruecos. Antiatlas: Sur le Dj. Kest, sol siliceux, $1500 \mathrm{~m}, 1-\mathrm{V}-1934$, nombre del recolector ininteligible, posiblemente Emberger, BC 84886; Cerca del Tizi N'Aït Iftene, sobre roquedo silíceo, $29^{\circ} 50^{\prime} \mathrm{N} 9^{\circ} 02^{\prime} \mathrm{W}, 29 \mathrm{RMP} 9600$, 1600 m, 8-IV-1998, Gómiz, Herb. F. Gómiz 5226; Tizi N'Aït Iftene, ladera silícea, $29^{\circ} 50^{\prime} \mathrm{N} 9^{\circ} 02^{\prime}$
W, 29RMP9600, 1600 m, 4-V-2000, Gómiz, Herb. F. Gómiz 5630.

\section{Cladanthus scariosus}

Marruecos. Alto Atlas: Goundafa, à Ijoukak, graviers de l'oued Agoundis, 1300 m, 28-IV1931, E. Jahandiez, Plantes marocaines No 239, BC 144105; Berges de la Réraïa à Asni, $1000 \mathrm{~m}$, 9-VI-1935, J. Gattefossé, BC 86417; Agadir. Environs $2 \mathrm{~km}$ avant le col du Tizi n'Test versant Taroudannt, rocailles calcaires, abondant, ca. $1900 \mathrm{~m}, 30^{\circ} 42^{\prime} \mathrm{N} 8^{\circ} 25^{\prime} \mathrm{W}, 30-\mathrm{V}-1980, A$. Charpin, J. Fdez. Casas, F. Jacquemoud \& D. Jeanmonod, MA 246670; $57 \mathrm{~km} \mathrm{~S}$ from Marrakech along unmade minor road to Oukaïmeden from Tahanaoute, at cross-roads, $1800 \mathrm{~m}, 31^{\circ} 15^{\prime} \mathrm{N} 7^{\circ} 51^{\prime} \mathrm{W}, 3-\mathrm{VII}-1987$, S. L. Jury, Moh. Rejdali, \& M. F. Watson, BC 688915 (ut Ormenis mixta), MA 391344 (ut Ormenis mixta); Province of Khénifra, road P-21 between Midelt and Er-Rachidia, Tizi-n-Talrhent, near track to Djebel Ali-ou-Rbeddou, oued, rocks, $1860 \mathrm{~m}, 32^{\circ} 35.647^{\prime} \mathrm{N} 4^{\circ} 32.230^{\prime} \mathrm{W}, 9-\mathrm{V}-1995$, R. Vogt \& Ch. Oberprieler, MA 586852; Unos 11 $\mathrm{km}$ al $\mathrm{N}$ de Taddert, en cuneta pedregosa silícea, $31^{\circ} 26^{\prime} 47^{\prime \prime} \mathrm{N} \mathrm{7} 7^{\circ} 25^{\prime} 00^{\prime \prime} \mathrm{W}, 29 \mathrm{RPQ} 5080,1400$ m, 5-VI-1996, Gómiz, Herb. F. Gómiz 4873; Road from Imilchil to Msemrir and Boumalne Dadès, $\mathrm{S}$ and $\mathrm{E}$ of Imilchil. Steep, SE-facing rocky bank, $2350 \mathrm{~m}, 31^{\circ} 59^{\prime} \mathrm{N} 5^{\circ} 34^{\prime} \mathrm{W}, 30 \mathrm{R} 2575993541805$, 8-VII-1997, S. L. Jury, A. Abaouz, M. Ait Lafkih \& A. J. K. Griffiths, MA 616334; Ait Said, 38 km from Boumalne along road to Msemrir above the Gorges du Dadès, dry bank below road, $1720 \mathrm{~m}$, $31^{\circ} 35^{\prime}$ N 5 5 53' W, 30R 2558773497107,10 VII-1997, S. L. Jury, A. Abaouz, M. Ait Lafkih \& A. J. K. Griffiths, MA 615582; S. from Marrakech, $3 \mathrm{~km}$ below Oukaïmeden on road to Vallée de l'Ourika and Marrakech. Steep rocky bank, $2380 \mathrm{~m}, 31^{\circ} 13^{\prime} \mathrm{N} 7^{\circ} 50^{\prime} \mathrm{W}, 29 \mathrm{R} 611632$ 3454887, 28-VII-1997, S. L. Jury, S. C. Darwin, A. J. K. Griffiths \& M. Rejdali, MA 614938; S. from Marrakech, 2,5 km below Oukaïmeden on road to Vallée de 1'Ourika and Marrakech. Sfacing vertical sandstone cliff in gorge, $2400 \mathrm{~m}$, 29R 610073 3454460, 28-VII-1997, S. L. Jury, S. C. Darwin, A. J. K. Griffiths \& M. Rejdali, MA 614936; Vertiente S del Tizi N Test, sobre roquedo húmedo, $30^{\circ} 52^{\prime} 04^{\prime \prime} \mathrm{N} 8^{\circ} 21$ ' 32 ' $\mathrm{W}$, 29RNQ6115, 1950 m, 4-VI-1998, Gómiz, Herb. 
F. Gómiz 5360; 5 km N en dessous du Tizi-nTichka, sur la route de Marrakech à Ouarzazate, surfaces surpâturées avec seulement quelques pieds d'Echinops et de Carlina, $2050 \mathrm{~m}, 31^{\circ} 19^{\prime}$ 50 " N 7 722 ' 02" W, 14-VIII-1999, D. Podlech, MA 692382. Alto Moulouya: Col du Larais, rocailles calcaires, $1800 \mathrm{~m}, 24-\mathrm{VI}-1924, E$. Jahandiez, Plantes marocaines $\mathrm{N}^{\circ} 705, \mathrm{BC} 77942$; Inmediaciones de Ait Oumghar, en terreno pedregoso estepario, $1560 \mathrm{~m}, 32^{\circ} 39^{\prime} 29^{\prime \prime} \mathrm{N}^{\circ}$ 57' 42" W, 30SUB1615, 18-V-2006, Gómiz, Herb. F. Gómiz 7462. Antiatlas: Vertiente S del Tizi n'Tazazert (J. Sarhro), en terreno rocoso eruptivo, $2225 \mathrm{~m}, 31^{\circ} 07^{\prime} 07^{\prime \prime} \mathrm{N} 5^{\circ} 46^{\prime} 40^{\prime \prime} \mathrm{W}$, 30RTV3546, 16-V-2006, Gómiz, Herb. F. Gómiz 7438 .

Las áreas de distribución de las otras dos especies, C. arabicus (L.) Cass. y C. mixtus (L.) Chev., ocupan, dentro de Marruecos, la práctica totalidad del territorio; extendiéndose, además, ampliamente fuera de sus límites (Oberprieler, 2002: 255; Vogt \& Oberprieler, 2002: 655; Bremer \& Humphries, 1993: 131; Jahandiez \& Maire, 1931-1934: 764).

AGRADECIMIENTOS. A Félix Llamas García, por la lectura crítica del manuscrito; y a los conservadores de los herbarios BC y MA, por las facilidades dadas para la consulta de los mismos.

\section{BIBLIOGRAFÍA}

BATTANDIER, J. A. -1921- Exploration botanique de la Haute Moulouya (Dr. Nain). Bull. Soc. Hist. Nat. Afrique N. 12: 6-11.

BENABID, A. -2000-Flore et écosystèmes du Maroc. Éditions Ibis Press. Paris; Librairie et éditions Kalila Wa Dimna. Rabat.

BENEDÍ, C. -1986- Nota sobre Chamaemelum Miller. Fontqueria 10: 1-4.

BENEDÍ, C. -1987- Revisió taxonòmica del gènere Chamaemelum Miller (Asteraceae) a la Península Ibèrica i les Illes Balears. Collect. Bot.(Barcelona) 17(1): 55-65.

BENEDÍ, C. -1988- Chamaemelum africani notulae criticae. Candollea 43: 123-127.

BREMER, K. \& C. J. HUMPHRIES -1993Generic monograph of the Asteraceae-
Anthemideae. Bull. nat. Hist. Mus. Lond. (Bot.) 23(2): 71-177.

CHARCO, J. -2001- Guía de los árboles y arbustos del norte de África. Agencia española de cooperación internacional. Madrid.

GUINEA, E. -1970- Santolina europaea. Anales Inst. Bot. A. J. Cavanilles 27: 29-44.

JAHANDIEZ, E. \& R. MAIRE -1931-1934Catalogue des Plantes du Maroc. Vols. 1-3. Alger.

LITARDIERE, R. de \& R. MAIRE -1930Contribution à l' étude de la flore du Maroc, 2. Mém. Soc. Sc. Nat. Maroc, 26: 1-56.

OBERPRIELER, C. -2002- A phylogenetic analysis of Chamaemelum Mill. (Compositae: Anthemidae) and related genera based upon nrDNA ITS and cpDNA trnL/trnF IGS sequence variation. Bot. J. Linn. Soc., 138: 255-273.

VOGT, R. \& C. OBERPRIELER -2002Chamaemelum y Cladanthus in Valdés, Rejdali, Achmal El Kadmiri, Jury \& Montserrat (eds.). Catalogue des plantes vasculaires du Nord du Maroc, incluant des clés d'identification. CSIC. Madrid.

Aceptado para su publicación en julio de 2006

Dirección del autor: Apartado 1007, E-24080 León, España.fgomiz@teleline.es 